\title{
A Dynamic Marking Scheme of Assured Service for Alleviating Unfairness among Service Level Agreements
}

\author{
Seung-Joon Seok ${ }^{1}$, Seok-Min Hong ${ }^{1}$, Sung-Hyuck Lee ${ }^{2}$, and Chul-Hee Kang ${ }^{1}$ \\ ${ }^{1}$ Department of Electronics Engineering, Korea University \\ 1, 5-ga, Anam-dong, Sungbuk-gu, Seoul 136-701 Korea \\ \{ssj, mickey, chkang\}@widecomm.korea.ac.kr \\ http://widecomm.korea.ac.kr/ ssj \\ ${ }^{2}$ Network Protocol T.G., i-Networking Lab., Samsung Advanced Institute of Technology \\ San 14-1, Nongseo-ri, Kiheung-eup, Yongin-shi, Kyungki-do, Korea \\ starsu@sait.samsung.co.kr
}

\begin{abstract}
Assured Service, which is a service model of the Internet Differentiated Services (DiffServ) architecture, is not currently well implemented on the Internet, mainly because TCP employs an AIMD (Additive Increase and Multiplicative Decrease) mechanism to control congestion. Many studies have shown that the current Assured Service model does not assure the target rate of high profile flows in the presence of numerous low profile flows, and does not equably distribute the reservation rate of an SLA among multiple flows included in the SLA. In this paper, the former problem is referred to as inter-SLA unfairness and the latter problem is referred to as intra-SLA unfairness. We propose a marking rule, called RAM (Rate Adaptive Marking) that simultaneously diminishes both of these unfairness problems. The RAM method marks sending packets, at a source node or at an edge router, in inverse proportion to throughput gain and in proportion to the reservation rate and throughput dynamics. Three experiments with the ns- 2 simulator are performed to evaluate the RAM scheme. The simulation results show that the RAM scheme may significantly reduce inter-SLA unfairness and the intra-SLA unfairness.
\end{abstract}

\section{Introduction}

An Assured Service (AS), as first defined in [1], is an example of an end-to-end service that can be built from the proposed differentiated services enhancements to IP using a single PHB of Assured Forwarding PHB. The basic concept of the AS is that packets are forwarded with high probability as long as the traffic from the customer site does not exceed the subscribed rate (traffic profile). Thus, it is necessary for the Assured Service that the interior router allocates some bandwidth and a buffer for each AF class, and that a user or user group of an Assured Service establishes a contract with the provider, which defines the profile for the service expected to be obtained; SLA (Service Level Agreement). This contract can be made only for a TCP flow, aggregated TCP flows or a combination of multi-protocol flows. The building blocks of the AS include a traffic marker at the edge router of a domain and a differ-

The original version of this chapter was revised: The copyright line was incorrect. This has been corrected. The Erratum to this chapter is available at DOI: 10.1007/978-3-540-45812-8_28 
entiated dropping algorithm in the network interior router. The traffic marker ranks packets as IN or OUT depending upon the SLA. An example of a differentiated dropping algorithm is RIO (RED with IN and OUT). The RIO algorithm uses the same RED algorithm for the IN packets and OUT packets, albeit with a different set of parameters for each. In particular, the OUT packets are preferentially dropped, upon evidence of congestion at the bottleneck, before the IN packets.

In previous studies, it was reported that there are two types of unfairness in the case of an Assured Service. Firstly, let us suppose that TCP flows with an Assured Service path through the same bottleneck link. In particular, let us assume that the TCP flows have different traffic profile rates. Under these conditions, the flows expect to share the excess bandwidth of the bottleneck link so as to meet their target rates. The target rate is the sum of the traffic profile rate plus an equal share of the excess bandwidth of the bottleneck link for all flows. This is called the equal sharing target rate [3]. However, several studies [4][5][6] have shown that TCP flows with a high profile rate and/or long round trip time (RTT) barely meet their target rate in the presence of numerous flows with low profile rate. The reason for this problem is that current TCP additively increases its window size and multiplicatively decreases it when any packet is lost. This causes flows with a high profile rate to take a longer time to reach to their target rates following packet loss, or not to reach to. For convenience, this problem is herein referred to as ì inter-SLA unfairnessî. Unfairness is defined as excess bandwidth of a bottleneck link not being distributed equably among flows that go through that link.

Next, the SLA may cover a set of flows. In this case, however, there exists unfair sharing of the reserved bandwidth or of the profile rate among the aggregated flows [7][8][9][10]. This unfairness between the aggregated flows is a serious problem in the Assured Service model. The unfairness can be caused by differences in RTTs, in link capacities, or in congestion levels experienced by flows within the network, though the total throughput of the aggregation still reaches the reservation rate. This type of unfairness is herein referred to as ìintra-SLA unfairnessî. To balance or fix the unfairness, a sophisticated marking mechanism is required to distribute the profile rate fairly to the aggregated flows at the edge routers [7][8][9][10].

Most studies of Assured Service focus on TCP protocol extensions [3][11], shaping [12][13] and dropping policies [3][14]. However, few approaches propose to change the current general marking rule which is that the total IN marking rate is equal to the reservation rate. In this paper, we present a new marking rule to resolve the two unfairness problems. The rest of the paper is organized as follows. Section 2 describes TCP dynamics in a differentiated services network, previous approaches to changing the general marking rule in order to alleviate inter-SLA unfairness and the axiom of the approach set out in this paper, called the Rate Adaptive Marking (RAM) scheme, in order to reduce the dynamics. Section 3 details the architectures and algorithms of the RAM. Section 4 studies the performance of RAM using an ns-2 network simulator and compares it with that of normal marking schemes and section 5 discusses some unresolved issues and concludes this paper. 


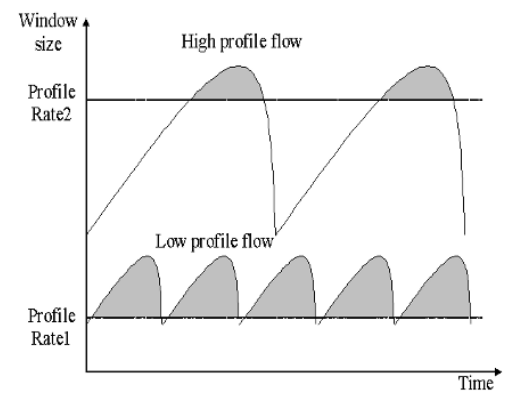

(a) general standard marking scheme

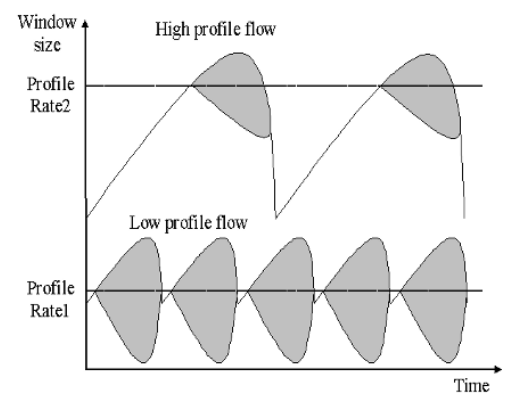

(c) Namís proportional marking scheme

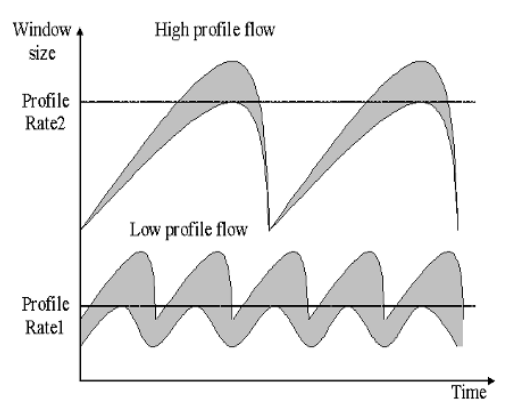

(b) Fengís soft bandwidth guarantee scheme

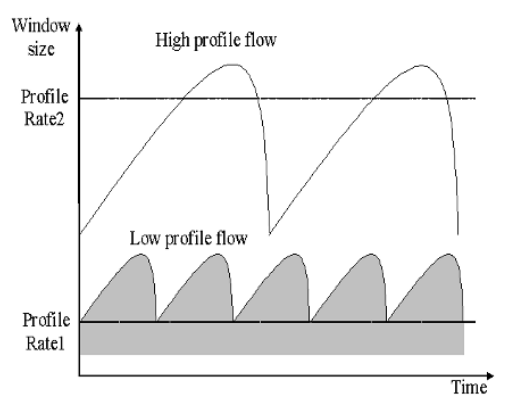

(d) proposed RAM scheme

Figure 1. Marking methods for two flows in the cases when a high profile flows and a low profile flows competes with each other at a bottleneck link, marking methods.

\section{Two Previous Works and Rate Adaptive Marching Scheme}

In the Assured Service architecture, it is generally assumed that sending packets are marked with IN as long as they do not exceed the reservation rate (traffic profile rate), otherwise marked OUT, since the marking rate is the same as the profile rate of the SLA. Figure 1(a) shows this general marking scheme for two flows having different reservation rates. In this figure, the shadowed region indicates the quantity of OUT marked packets. So far, there are few solutions that approach the problem from the point of view of changing this general marking rule. In this section, we describe the two approaches which do so, and then propose a new approach, called rate adaptive marking.

\subsection{Previous Approaches}

Wu Cheng Feng [15] proposed a ì soft bandwidth guaranteeî scheme that determines the marking rate adaptively according to a flowís throughput. In this scheme, by de- 
fault, all packets are generated as low priority packets. If however, the observed throughput falls below the minimum requested rate, the marking mechanism starts prioritizing packets until the desired rate is reached. He considered that this scheme was carried out at the edge node or the source node. The first was referred to as source transparent marking and the second was referred to as source integrated marking. In particular, the second scheme considered the integration of the marking mechanism and the TCP control mechanism. Figure 4(b) shows marking method of Fengís scheme. Fengís scheme considers the reservation rate to be allocated to a flow as a target rate. Thus, it can not provide flows with equal share of excess bandwidth, in the case where network is lightly loaded.

Nam et al. [16] use a proportional marking method in which low profile flows have more OUT marked packets and, therefore, more dropped packets. In the proportional marking scheme, when a packet arrives, a meter calculates the average rate. If this average rate exceeds the flowís target rate, the proportional marking method marks the code point of the packet as OUT in proportion to the difference between the average rate and the target rate. Figure 4(c) shows the marking method of Namís scheme. As figure 4(c) shows, the high profile flow has a marking rate which is lower than its reservation rate, following a situation in which temporary throughput is over the reservation rate. This can not help the fairness, though decreasing the marking rate of the low profile may help.

In this paper, another marking scheme, called Rate Adaptive Marking (RAM), is proposed. The two previous schemes considered only inter-SLA unfairness, while the RAM scheme can alleviate both inter-SLA unfairness and intra-SLA unfairness. The details of this scheme are described in the next two sections.

\subsection{The Axiom of Rate Adaptive Marking}

What is the Rate Adaptive Marking (RAM)? This subsection describes the conceptual and operational mechanisms of this scheme. A RAM marker determines a flowís marking rates periodically, depending on information about throughput rates collected during a previous period, and marks sending packets according to this rate during the next period. The basic principle of RAM strategy is marking packets as IN in inverse proportion to the throughput gain and in proportion to the reservation rate (equation1, 2). The gain denotes how much the throughput of a flow is over its reservation rate. Also, RAM makes a flowís IN marking rate to be proportional to the dynamics in that flowís throughput (equation 3). Unless otherwise stated, a simple marking rate denotes the IN marking rate.

$$
\begin{aligned}
& \frac{1}{\text { throughput_gain }} \propto \text { marking_rate } \\
& \text { reservation_rate } \propto \text { marking_rate }
\end{aligned}
$$

throughput_dynamics $\propto$ marking_rate 
Let us assume that two flows with the same reservation rate have different throughputs. In the case of RAM, the flow that gets the higher throughput is given a lower marking rate than the flow with lower throughput. This marking strategy causes that one flow to experience packet loss earlier than the other, when next congestion arises. Therefore, the gap between the two throughputs is reduced. Whereas, in the normal marking strategy, the two flows are given identical marking rates, because the marking rate of a flow is generally the same as its reservation rate. Next, let us assume another case where there are two flows with different reservation rates: a high profile flow and a low profile flow. The difference in reservation rate is the most important reason leading to inter-SLA unfairness. The high profile flow takes a longer time to reach the temporal throughput just before a packet loss, because the congestion window size is reduced more in the high profile flow than in the low profile flow. In other words, the high profile flow has larger throughput dynamics, between the maximum temporal throughput and the average throughput (or minimum temporal throughput), than the low profile flow. Thus, in this case, RAM tries to render the marking rate proportional to the throughput dynamics.

The RAM marker maintains state information of all flows that pass through it, and periodically determines the marking rate for each flow according to the following RAM rule (equation 4). The RAM marker monitors two throughput levels for all flows; temporal throughput and long term average throughput. The temporal throughput is an average throughput for a short term interval such as an average RTT, while the long-term average throughput is based on a relative long period of time such as the congestion interval. The following equation is the RAMís rule for determining the marking rate.

$$
\text { marking_rate }=\frac{(\text { Th } \max -\text { Thavg })}{(\text { Th } \max -\text { RsvRate })} \times \text { RsvRate }
$$

where Thmax is the maximum temporal throughput during the immediately preceding congestion interval and Thavg is the long term average throughput of the flow during the congestion interval. This equation connotes the basic principles of the RAM scheme. In the right-hand side of the equation, the ì Thmax-Thavgî term denotes the flowís throughput dynamics and ì(Thmax-RsvRate)/(Thmax-Thavg)î the flowís throughput gain. Finally ì $R s v R a t e \hat{~ i s ~ t h e ~ f l o w i ́ s ~ r e s e r v a t i o n ~ r a t e . ~}$

If a flow achieves an average throughput that is equal to its reservation rate (RsvRate) in the RAM scheme, the marking rate becomes the reservation rate. Also, if the average throughput is over the reservation rate, the marking rate will be lower than the reservation rate and proportional to the gap between the average throughput and the reservation rate. In contrast, if the average throughput is below the reservation rate, the marking rate will be higher than the reservation rate and also inversely proportional to the gap. However, it can happen that the result of equation 4 is negative or has an infinite value, when Thmax is below or equal to the RsvRate. This case denotes that the throughput does not reach its reservation rate as well as its target rate. The RAM scheme has an upper bound and a lower bound for the marking rate, in order to protect the marking rate and the throughput from experiencing heavy fluctuations. The upper bound is considered to be several times the reservation rate (i $\alpha \times R s v R a t e \hat{i}$ ) and the lower bound to be a half or less of the reservation rate 
(" $\beta \times R$ RvRate"). So the marking rate is set to the upper bound, if the result of equation 4 is over the upper bound or negative, and the lower bound, if the result is positive and under the lower bound. Thus, the equation 4 can be re-written as follows.

$$
\text { marking_rate }=\min \left(\alpha, \max \left(\beta,\left(\frac{\text { Th } \max -\text { Thavg }}{\text { Th } \max -\text { RsvRate }}\right)\right)\right) \times \text { RsvRate }
$$

This RAM strategy supports various network environments and, in particular, various network congestion levels. If the network is over-subscribed or the flows are under-provisioned, the average throughputs of the flows or maximum temporal throughputs (Thmax) may be below their reservation rates and so the marking rates of the flows are much higher than the corresponding reservation rates. In this case, almost all packets are marked as IN (high precedence) and packet losses are proportional to the flow's marking rate. As a result, the flow's throughput is proportional to the reservation rate. On the other hand, if the flows are over-provisioned or the network is under-subscribed, all flows have an average throughput superior to their reservation rates and almost packets are marked as OUT (low precedence). This case is almost equal to the best-effort case, because the aggregation of the reservation rates is insignificant amount in comparison to the bottleneck link bandwidth. Therefore, all flows come to have similar excess bandwidth and so to have similar average throughputs.

Consider the case of a non-responsive flow using UDP or a flow with low dynamics in throughput, the gap between Thmax and Thavg is very small. Thus, the value of equation 4 approaches zero, if the flow is not in an under-provisioned state. Also, the marking rate becomes the lower bound, because the value is under the lower bound and positive. If the flow is over-provisioned, however, the marking rate is increased over its reservation rate because Thmax is less than RsvRate. This operation can reduce the impact of non-responsive flows (UDP) on responsive flow (TCP). Figure 1(d) shows the marking method of the RAM strategy, in which the high profile flow is marked more aggressively than in the other methods (shown in figure 1), but the low profile flow is marked less aggressively.

If a marker treats a single corresponding flow, then this marker can be located in a flow source or an edge router. The former case is referred to as source marking and the latter case as edge marking. An important feature of source marking is that the RAM marker can monitor the throughput accurately. This is because the ACK information can be provided to the marker. However, it is impossible for edge marking to estimate the throughput accurately, because of the absence of the ACK information. Thus, the edge marking RAM uses the sending rate of the flow source to measure the performance of the flow. In the source marking case, the marking rate is updated whenever packet loss is detected. The maximum temporal throughput can be derived from the congestion window size at that time, because the congestion window is maximized just before congestion control backs it off. The average throughput also denotes the average acknowledged traffic between the previous congestion point and this congestion point (congestion period). However, the RAM marker that is located in the edge router can not detect the flow's packet losses. Thus, the edge marker has two time intervals to implement for the RAM scheme. Every average round trip time, the edge marker calculates the temporal throughput as the average sending rate for the 
round trip time and compares this with previous ones, in order to determine the maximum value. Also, every average congestion interval, the average sending rate for the interval is calculated. Other behaviors of this marking scheme are the same as that of the source marking RAM scheme.

It is more general that an SLA includes multiple flows and a single traffic profile of aggregated flows. In this case, a marker must be located at the edge router to support multiple flows from different sources. The general marking scheme does not maintain each flow state and marks packets as IN, in proportion to the sending rates of the flows. The RAM scheme should also be carried out at the edge router. The RAM marker generates a RAM instance corresponding to each flow. This RAM instance determines the marking rate depending on RAM strategy (equation 5) and marks packets according to this marking rate. The Reservation rate of each flow is considered as the traffic profile rate for aggregated flows / the number of flows. The

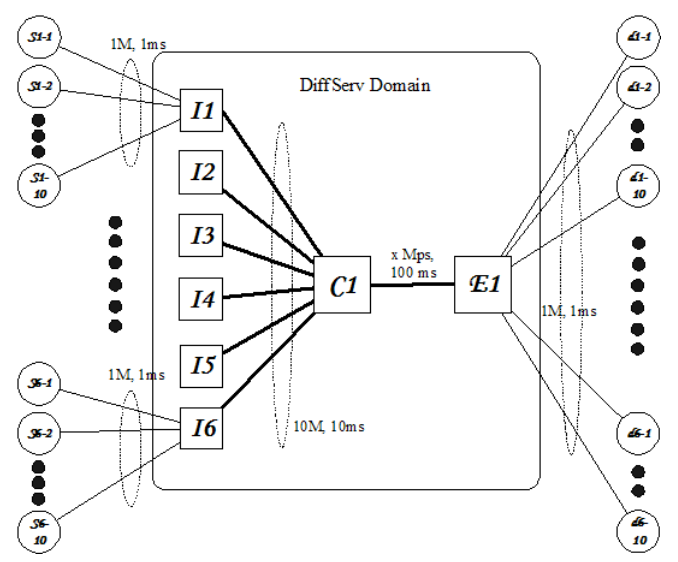

Figure 2. Experimental topology used to study inter-SLA unfairness

Table 1. Simulation parameters for evaluation of interSLA unfairness

\begin{tabular}{|c|c|}
\hline Parameters & Value \\
\hline S-I link bandwidth & $1 \mathrm{Mbps}$ \\
\hline S-I link delay & $1 \mathrm{~ms}$ \\
\hline I-C link bandwidth & $10 \mathrm{Mbps}$ \\
\hline I-C link delay & $10 \mathrm{~ms}$ \\
\hline C-E link bandwidth & $5,6.3,7,8$, and $9 \mathrm{Mbps}$ \\
\hline C-E link delay & $100 \mathrm{~ms}$ \\
\hline E-D link bandwidth & $1 \mathrm{Mbps}$ \\
\hline E-D link delay & $1 \mathrm{~ms}$ \\
\hline Router buffer size & 100 packets \\
\hline Packet size & 1000 byte \\
\hline RIO parameter 1 (IN) & $50 / 80 / 0.02$ \\
\hline RIO parameter 2 (OUT) & $10 / 40 / 0.5$ \\
\hline Simulation time & $200 \mathrm{~s}$ \\
\hline
\end{tabular}


marking algorithm for each flow is the same as the edge marking scheme of single flow. In particular, this scheme also uses the sending rate of each flow as information to determine the marking rate of the flow.

\section{Performance Study}

In order to confirm the performance effect of the RAM scheme as against previous approaches, two experiments have been performed, using ns-2 [20], on several simulation models. The first experiment is the evaluation of inter-SLA unfairness through the comparison of the RAM with Fengís soft bandwidth guarantee, Namís proportional marking and the general marking scheme and the second experiment tests the intra-SLA unfairness using the RAM scheme, the general marking, and equally sharing scheme.

\subsection{Evaluation of Inter-SLA Unfairness}

We first examine the effectiveness of the RAM scheme to alleviate the inter-SLA unfairness when there are only TCP flows. Both RAM scheme types, source marking RAM and edge marking RAM, are considered in this experiment. Figure 2 depicts the

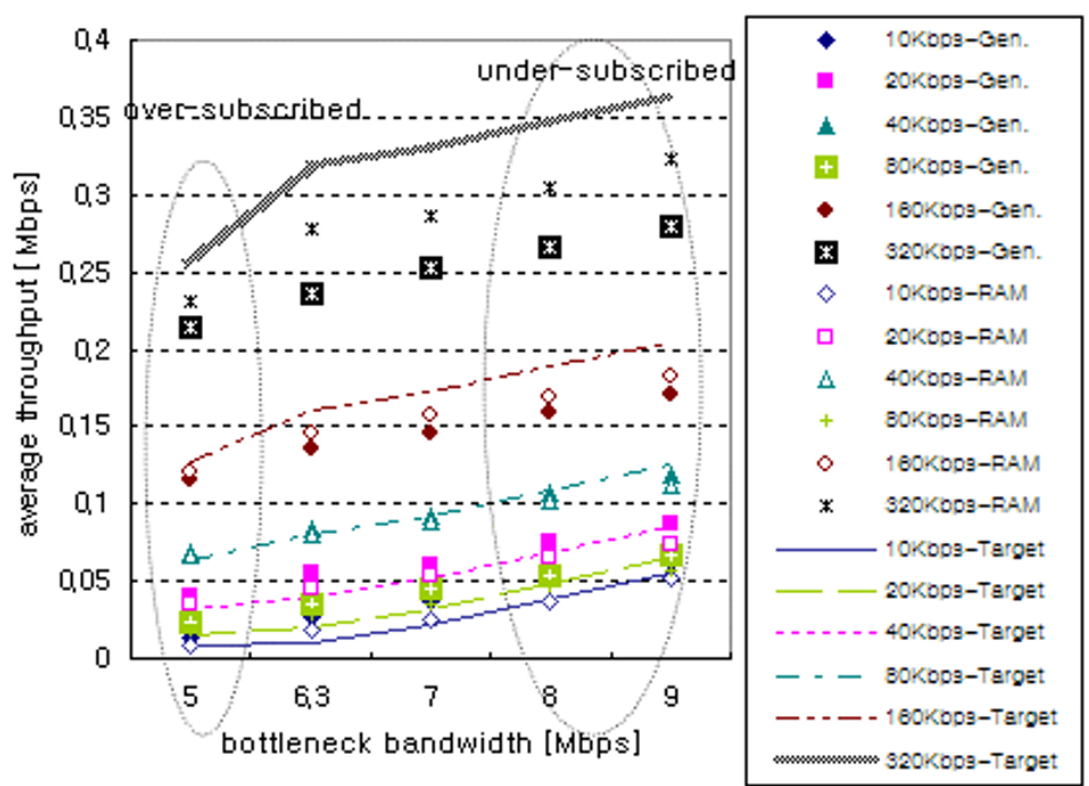

Figure 3. Average Throughputs of TCP flows using RAM implemented in TCP source

solid line : target rate (Target) and using normal marking

filled item : average throughput using general marking (Gen.) empty item : average throughput using source marking RAM (RAM) 
testbed topology for this experiment. In this topology, there are 60 TCP flows and each edge router is connected with 10 TCP sources. The profile rates for each TCP flow of s1-x, s2-x, s3-x, s4-x, s5-x and s6-x are 0.01Mbps, 0.02Mbps, 0.04Mbps, $0.08 \mathrm{Mbps}, 0.16 \mathrm{Mbps}$ and $0.32 \mathrm{Mbps}$, respectively. All flows are routed through a common bottleneck link C1-E1 to model the competition for the excess bandwidth. In this way, the total reservation rate of the bottleneck link is 6.3Mbps. Also, this experiment considers several network environments in which different bottleneck link bandwidths, $x=5,6.3,7,8$, and 9Mbps, are used. Other parameters of the testbed are represented in table 1 .

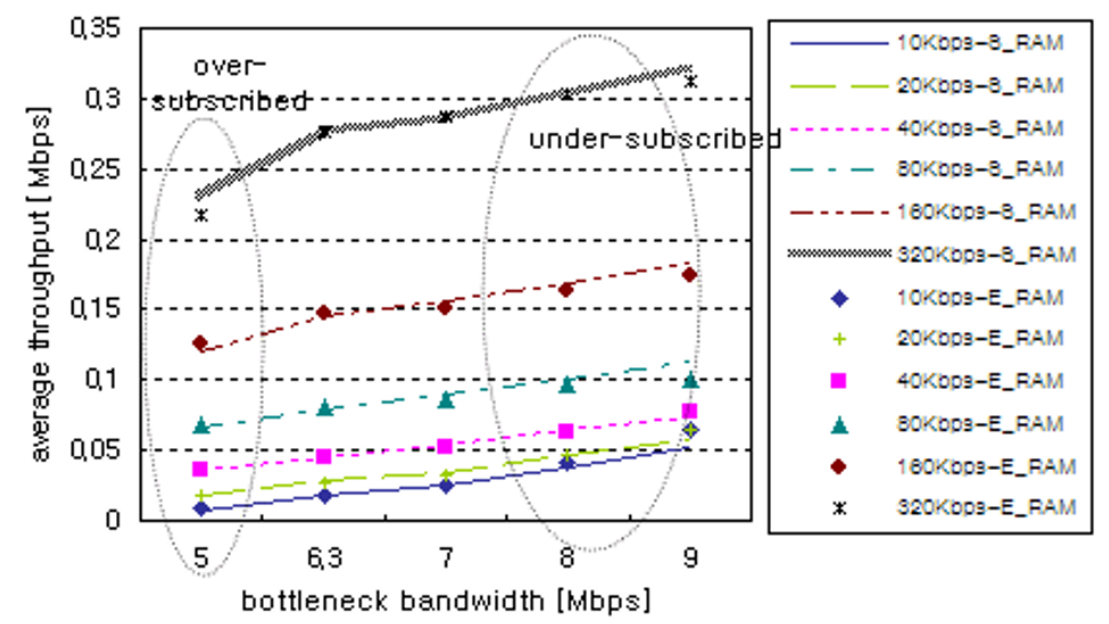

Figure 4. Average Throughputs of TCP flows using RAM implemented in TCP source and in edge router

solid line : RAM scheme in source (S_RAM)

item : RAM scheme in edge router (E_RAM)

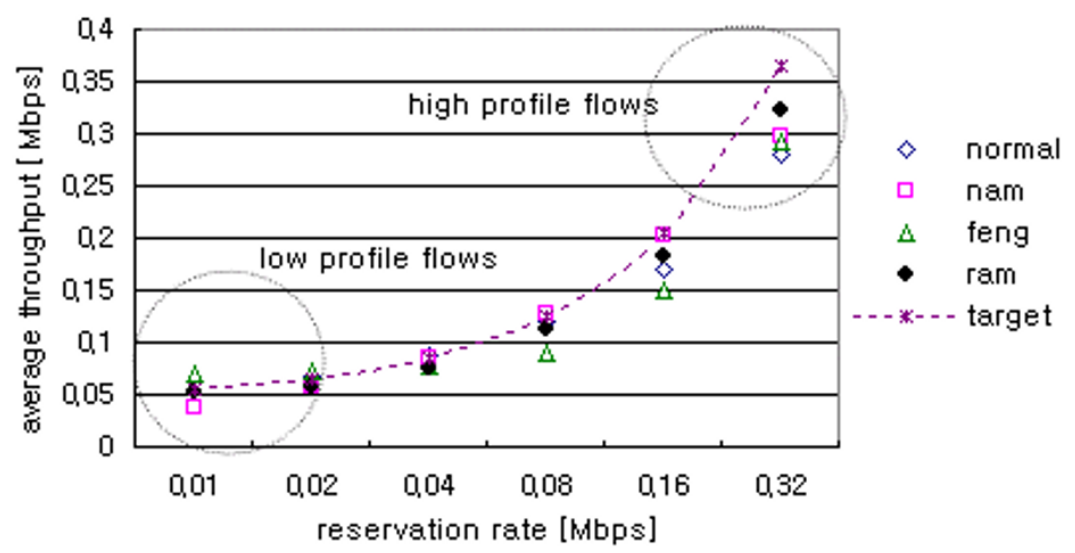

Figure 5. Comparison of effectiveness of marking schemes in the case when bottleneck link bandwidth is $9 \mathrm{Mbps}$. 
Figure 3 shows the average throughputs of the 10 TCP flows with the same reservation rate when the source marking RAM rule is applied and when the general marking rule is applied. These throughputs are compared with the ideal target rates. It is impossible for all average throughputs to be at the ideal target rates. It is required, however, for all average throughputs to be fairly near to their target rates. When the general marking rule is used, low profile $(0.01,0.02,0.04$, or $0.08 \mathrm{Mbps})$ flows achieve throughputs that are near or over their target rates, regardless of network load. Also, these throughputs are higher than those obtained when the source marking RAM rule is used. When the general marking is applied, however, the throughputs of the high profile $(0.08,0.16$, or $0.32 \mathrm{Mbps})$ flows are less than those obtained when the source marking RAM rule is used, as well as being much less than the target rates. This problem can be ignored in the over-subscribed case, but is made worse as the network becomes under-loaded. However the RAM scheme can alleviate this problem. In figure 4, the source marking RAM rule is compared with the edge marking RAM rule. From this figure, we can see that the edge marking RAM technique can remedy the above problem as well as the source marking RAM technique does, because the result of the simulation using the edge marking RAM rule is similar to that of the source marking RAM rule, regardless of network load. Next, we compare the source marking RAM technique with Namís scheme, Fengís scheme and the general marking scheme, when the bottleneck link is well-provisioned (9Mbps). Figure 5 shows that Namís scheme gives the 40,80 , and 160 Kbps profile flows the maximum throughput. For the $320 \mathrm{Kbps}$ profile flow, however, the RAM scheme is optimal. Because inter-SLA unfairness is mainly impacted by the degradation of the $320 \mathrm{Kbps}$ flows (figure 3), improving the throughputs of these flows is the key factor for resolving this problem.

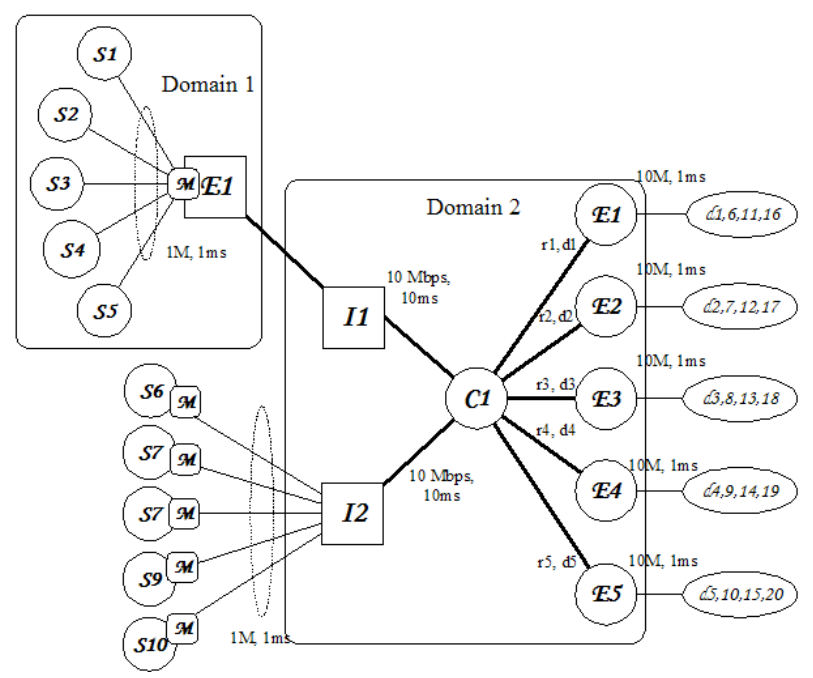

Figure 6. Experimental topology used to study the intra-SLA unfairness 
Table 2. Simulation parameters for evaluation of intra-SLA unfairness

\begin{tabular}{|c|c|}
\hline Parameters & Value \\
\hline S-I or E-I link bandwidth & $1 \mathrm{Mbps}$ \\
\hline S-I or E-I link delay & $1 \mathrm{~ms}$ \\
\hline I-C link bandwidth & $10 \mathrm{Mbps}$ \\
\hline I-C link delay & $10 \mathrm{~ms}$ \\
\hline $\begin{array}{c}\text { C-E link bandwidth } \\
\text { (r1, r2, r3, r4, r5) }\end{array}$ & $500 / 300 \mathrm{Kbps}$ \\
\hline $\begin{array}{c}\text { C-E link delay } \\
\text { d1, d2, d3, d4, d5) }\end{array}$ & $100 \mathrm{~ms}$ \\
\hline E-D link bandwidth & $10 \mathrm{Mbps}$ \\
\hline E-D link delay & $1 \mathrm{~ms}$ \\
\hline Router buffer size & $100 \mathrm{packets}$ \\
\hline Packet size & $1000 \mathrm{byte}$ \\
\hline RIO parameter 1 (IN) & $50 / 80 / 0.02$ \\
\hline RIO parameter 2 (OUT) & $10 / 40 / 0.5$ \\
\hline Simulation time & $200 \mathrm{~s}$ \\
\hline
\end{tabular}

\subsection{Evaluation of Intra-SLA Unfairness}

In this experiment, we consider the case in which an SLA includes multiple flows. Figure 6 depicts the experimental topology used to test the intra-SLA unfairness. In this topology, domain 1 and domain 2 establish an SLA, in which 500Kbps is allocated as the traffic profile of the aggregated flows from sources (s1, s2, s3, s4 and s5). Thus, these flows should be remarked at the E1 according to the SLA. The other flows are used for background traffic and assumed to be coming into domain 2 from different domains, except for domain1. The background sources (s6, s7, s8, s9, and s10) have different marking rates $(10,100,200,300$, and $400 \mathrm{Kbps}$ respectively). In order that each flow included in the SLA experiences different network status, flows1, 2, 3, 4 and 5 are routed through different routes with different congestion levels within domain 2. The congestion levels are controlled by the background flows. Other simulation parameters are represented in table 2. In the ideal case, each flow included in an SLA has a throughput and Jainís fairness index becomes one. As unfairness among the flows increase, the fairness index value decreases.

In this experiment, we carried out two simulations. In the first simulation, CBR streams are considered as background traffic, and in the second simulation, TCP streams are considered. We monitored the average throughputs of TCP flows included in the SLA, in the case when general marking, RAM-edge marking, or equal sharing marking (Sharing) is used. The general marking scheme marks incoming packets in proportion to the incoming rate of each flow. In equal sharing marking, however, the SLAís reservation rate is distributed equally among aggregated TCP flows. Figure 7 shows the simulation results. In first simulation result (figure 7(a)), the fairness index value of the general, RAM, and Sharing scheme are about 0.88 , 0.95 , and 0.93 , respectively. In the second simulation result (figure 7(b)), these values are $0.93,0.98$, and 0.97 , respectively. From these results, we confirmed that the RAM scheme can reduce intra-SLA unfairness as well as inter-SLA unfairness. 


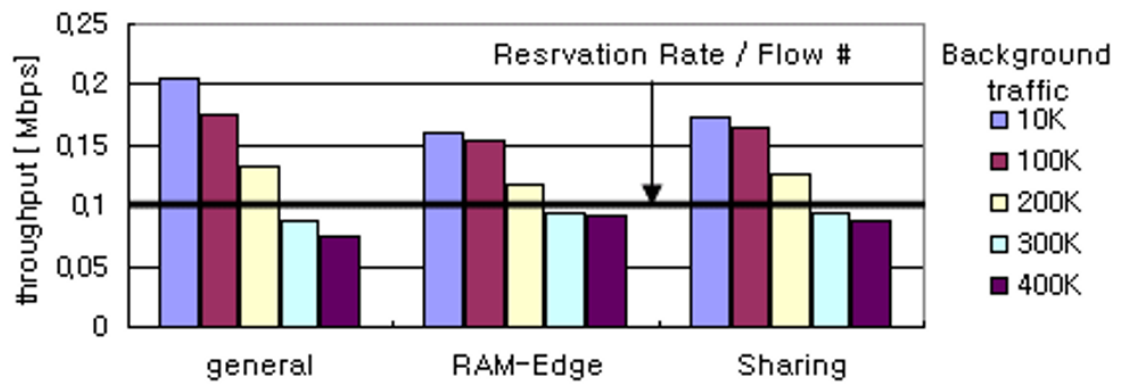

(a) In the environment in which background traffic is CBR stream, all core-egress link bandwidth is $0.5 \mathrm{Mbps}$.

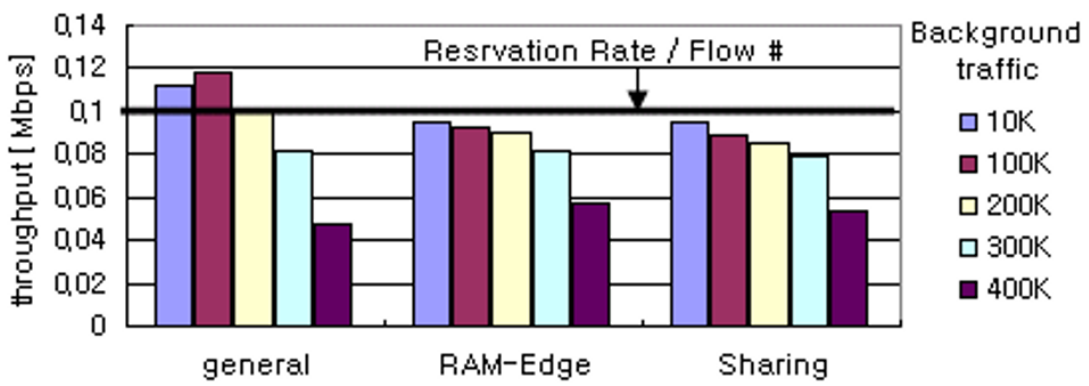

In the environment in which background traffic is TCP stream, all core-egress link bandwidth is $0.35 \mathrm{Mbps}$

Figure 7. Average Throughputs of TCP flows included in a SLA

\section{Conclusion}

In this paper, we described two serious problems, inter-SLA unfairness and intraSLA unfairness, which arise when providing an Assured Service and we discussed previous reports on this topic. We proposed an approach which consists of a modified marking rule, called RAM (Rate Adaptive Marking), for the Assured Service, to alleviate both unfairness problems. The RAM scheme determines a flowís marking rate periodically, in inverse proportion to throughput gain, and in proportion to throughput dynamics and to the reservation rate during a previous time period. We described two scenarios to implement the RAM scheme, source marking RAM and edge marking RAM. The source marking RAM scheme cooperates with TCP congestion control to calculate throughputs accurately. The edge marking RAM scheme operates at an ingress edge router and exploits the sending rate of each flow, instead of the throughput. The edge marking RAM scheme can support the marking of aggregated flows, whereas the source marking RAM scheme can not. The effectiveness of RAM was evaluated through computer simulations. The simulation results showed that the RAM technique can alleviate both inter-SLA unfairness and intra-SLA unfairness simultaneously. 


\section{References}

1. Heinanen, J., Baker, F., Weiss, W., and Wroclawski, J.: Assured Forwarding PHB Group. RFC2597, June (1999)

2. Blake, S., Black, D., Carlson, M., Davies, E., Wang, Z., Weiss, W.: An architecture for differentiated services. RFC 2598, June(1999)

3. Yeom, I., Reddy, A. L. N.: Realizing Throughput Guarantees in a Differentiated Service Network. In Proc. ICMCSí99, June (1999) 372-376

4. Rezende, J. F.: Assured Service Evaluation. In Proc. GLOBECOMí99, June (1999) 100-104

5. Seddigh, N., Nandy, B., Pieda, P.: Bandwidth Assurance Issues for TCP flows in a Differentiated Services Network. In Proc. GLOBECOMí99, June (1999) 1792-1798.

6. Ibanez, J. Nichols, K.: Preliminary Simulation Evaluation of an Assured Service. Internet Draft, Aug.(1998)

7. Yeom, I., Reddy, A. L. N.: Impact of marking strategy on aggregated flows in a differentiated services network. In Proc. IWQoS 1999 , May(1999)

8. Kim, H.: A Fair Marker. Internet Draft, April(1999)

9. Andrikopoulos, L., Pavlou, G.: A fair traffic conditioner for the assured service in a differentiated service Internet. In Proc. IEEE ICC 2000, June(2000)

10. Azeem, F., Rao, A., Kalyanaraman, S.: TCP-friendly traffic marker for IP differentiated services, In Proc. IWQoS'2000, June(2000) 35-48

11. Fang, W., Perterson, L.: TCP mechanisms for a diff-serv architecture, Tec. Rep

12. Cnodder, S. D., Elloumi, O., Pauwels, K.,: Rate adaptive shaping for the efficient transport of data traffic in diffserv networks. Computer Networks, Vol. 35(2001) 263-285

13. Li, N., Borrego, M., Li, S.: A rate regulating traffic conditioner for supporting TCP over Diffserv. Computer Commnications, Vol. 23(2000) 1349-1362

14. Park, W. H., Bahk, S., Kim, H.: A modified RIO algorithm that alleviates the bandwidth skew problem in Internet Differentiated Service. In Proc. ICCí00 (2000) 1599-1603

15. Feng, W., Kandlur, D., Saha, D., Shin, K.: Adaptive Packet Marking for Providing Differentiated Services in the Internet. In Proc. ICNPí98, Austin, TX, Oct. (1998) 108-117

16. Nam, D-H., Choi, Y-S. Kim, B-C., and Cho, Y-Z.: A traffic conditioning and buffer management scheme for fairness in differentiated services. in proc. ICATM 2001, April (2001)

17. Baines, M.: Using TCP Model To Understand Bandwidth Assurance in a Differentiated Services Network, In proc. IEEE Infocomm 2001 (2001)1800-1805

18. Padyhe, J., Firoiu, V., Townsley, D., Kurose, J.: Modeling TCP Throughput: A Simple Model and its Empirical Validation. CMPSCI Technical Report TR 98-008, University of Massachussetts, MA (1999)

19. Lee, S-H., Seok, S-J., Lee, S-J., Kang, C-H.: A Study of TCP and UDP flows in a Differentiated Services Network using Two Markers System. In proc. MMNS 2001, Oct. (2001) 198-203

20. Network Simulator ñ NS (version 2), http://www-mash.ca.berkely.edu/ns/ 